\title{
Automated detection of breast asymmetries
}

\section{Peter Miller and Sue Astley}

\author{
Department of Medical Biophysics, \\ University of Manchester \\ pim@wiau.mb.man.ac.uk
}

\begin{abstract}
Breast asymmetry is an important radiological sign of cancer. This paper describes the first approach aiming to detect all types of asymmetry; previous asymmetrybased research has been focussed on the detection of mass lesions. The conventional approach is to search for brightness or texture differences between corresponding locations on left and right breast images. Due to the difficulty in accurately identifying corresponding locations, asymmetry cues generated in this way are insufficiently specific to be used as prompts for small and subtle abnormalities in a computer-aided diagnosis system. We have undertaken studies to discover more about the visual cues utilized by radiologists. As a result, we propose a new automatic method for detecting asymmetry based on the comparison of corresponding anatomical structures, identified by an automatic segmentation of breast tissue types. We describe methods for comparing the shape and brightness distribution of these regions, and we present results obtained by combining evidence for asymmetry.
\end{abstract}

\section{Introduction}

Breast screening programmes have been introduced in many countries, with the aim of detecting cancer at an early stage in asymptomatic women. In Britain, for example, it is estimated that 1 in 12 women will be affected by breast cancer at some point in their lives. Screening generates a large number of mammograms requiring interpretation, and a variety of computer-based aids have been proposed to improve the performance of radiologists searching for small, subtle and infrequent abnormalities. One viable application of current image processing technology would be a system for prompting radiologists by indicating to them any suspicious regions.

There is a wide variation in the appearance of mammograms from different women, but normal left and right mammograms from the same woman are generally symmetric; radiologists consider asymmetry an important sign of abnormality. Masses are the most common asymmetric sign of cancer, and appear brighter than surrounding tissue, often with discernible margins. When a mass is obscured by other structures, it will usually be seen as a focal, though poorly defined, asymmetric density. A disturbance in the normally symmetrical flow of structures towards the nipple may also be indicative of cancer, which can have the effect of pulling structures in towards a point. This sign is particularly important in screening of asymptomatic women, when masses may be very small or barely visible.

\section{Automated detection of asymmetry}

Several investigators have used asymmetry in computerized schemes for the detection of mammographic masses. More subtle types of asymmetry - focal density and 
architectural distortion - have not previously been tackled using automated methods. Firstly, digitised left and right mammograms are registered by matching the breast boundaries extracted from each image. This creates a mapping between the two images, which enables features from corresponding locations in the left and right breast to be compared. The resulting map of local asymmetries is processed to remove spurious responses and to determine the most suspicious regions.

The methods differ mostly in their choice of image features for the measurement of local asymmetry. Yin et al [1] used brightness; Kimme et al [2] and Lau and Bischof [3] used brightness and texture; and Hand et al [4] used brightness, texture and shape. The degree of difference between corresponding feature values and the size and shape of the detected asymmetric region are used to decide if a region is suspicious. If only a few regions on each mammogram satisfy these criteria, they could then be indicated to a radiologist for final diagnosis.

Conventional methods are based upon the assumption that breast asymmetry is adequately represented by image asymmetry, once the left and right breast images have been registered. Unfortunately, there are many factors contributing to image asymmetry which have no radiological importance. Although the anatomy of normal left and right breasts is largely symmetric, several asymmetric factors are inherent in the process of mammographic examination. For instance, the amount and angle of compression applied to each breast will determine the position, size, density and brightness of structures on the mammogram [5]. Moreover, these false asymmetries often outweigh and outnumber any true abnormalities which may be present on the mammogram. This explains the need for the supplementary tests mentioned above, as the true abnormalities must be differentiated from the many false cues. Any improvement in specificity is likely to degrade sensitivity, leaving small, subtle cancers undetected. We therefore believe that conventional methods are limited to the detection of conspicuous mass lesions, a task which radiologists are themselves capable of performing to an acceptable standard. A successful prompting system for breast screening must also recognize small masses and architectural distortion, which may be the only asymmetric signs of early cancer, and are difficult for radiologists to detect.

\section{How do radiologists detect asymmetry?}

Our basic understanding of the nature and mammographic appearance of breast asymmetry has been gained from the radiological literature and in detailed discussions with experienced breast radiologists. It is apparent that several levels of symmetry are considered in mammographic interpretation. Firstly, an impression of overall symmetry of the whole breast and gland tissue is gained by viewing the images at a distance. Then each quadrant of the two breasts is compared, looking for architectural disturbance and localized increases in density [6]. Some experts suggest moving masks slowly across both mammograms, to ensure that no asymmetries are missed [7]. Many additional techniques are used to evaluate any suspicious asymmetries in more detail.

It is also noticeable that much of radiologists' reasoning is performed on a regional basis. As well as the four quadrants already mentioned, the region containing all of the gland tissue, called the gland disc, is an important feature in the comparison. This is because cancers which attack mainly fatty tissue or supporting structures, form 

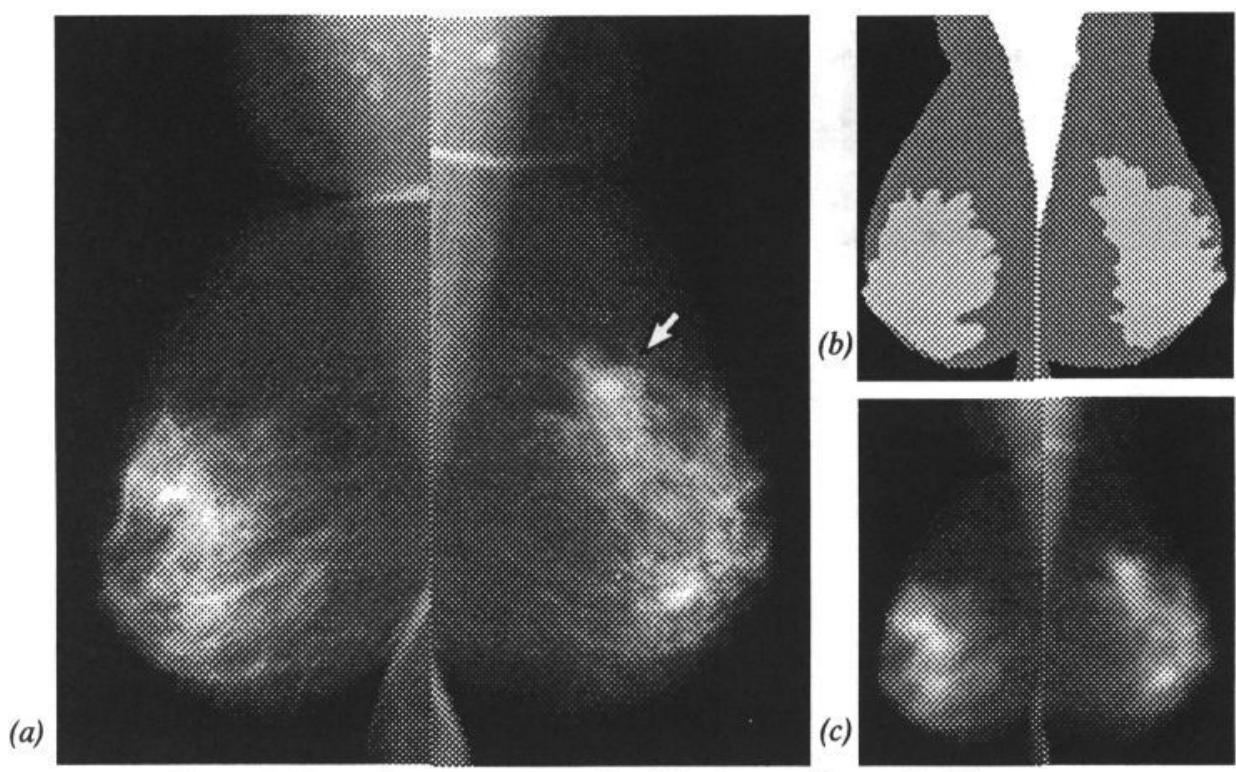

Figure 1. Example mammogram (a) with abnormality marked by arrow

(b) in shape study (c) in density study

bright, isolated foreground regions, and are relatively easy to detect on the darker background. The majority of cancers attack gland tissue, and can be obscured by other bright structures in the gland disc, so radiologists must also compare the shape and density of the gland discs to detect any underlying disease.

In summary, we believe that radiologists compare anatomically similar regions, independently of their precise location on the mammograms. This is in contrast with conventional automated methods, which are dependent on similar structures lying in corresponding locations. To test our hypothesis, and to discover which visual cues are used in the comparison of regions, we have employed a series of experiments to test radiologists [10].

A consultant breast radiologists selected 30 screening mammogram pairs, of which twelve contained abnormalities which were visible as varying degrees of asymmetry. In our first experiment the mammograms were processed to remove all brightness information, by superimposing radiologists' annotations of non-fat regions onto silhouettes of each breast. On most mammograms the gland disc was the only non-fat region (excluding the pectoral muscle), though two of the abnormal cases also contained isolated mass lesions. These data were designed to test if asymmetry could be detected using just shape and size cues from the non-fat regions. In the second experiment the mammograms were blurred to remove structural details and texture, leaving only brightness information. Figure 1 shows an abnormal mammogram pair, together with processed versions for the shape and density experiments.

We conducted a pilot experiment with three breast radiologists which showed that $60 \%$ of abnormal cases could be detected using shape information only. This result provides initial evidence for our assertion that asymmetry is detected using a comparison of regions. The shape and density experiments have been distributed to 30 breast 


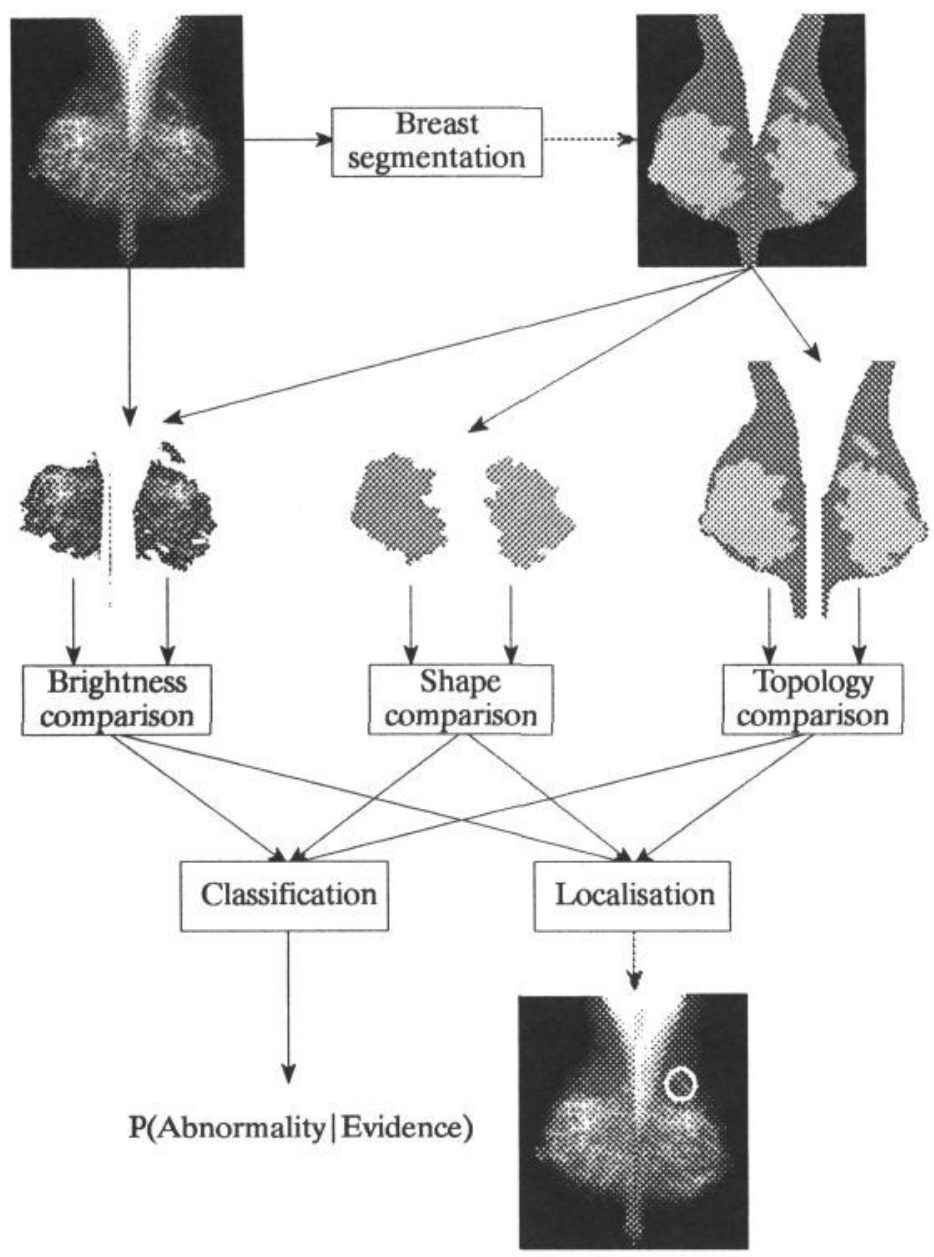

Figure 2. Schematic diagram of automated system for detecting asymmetry using anatomical features

radiologists around the country, and analysis of these results will allow us to measure and compare the radiological importance of each type of visual cue.

\section{A new automated approach}

In our discussion of radiologists' technique, we made the assertion that asymmetry is detected by comparing anatomically similar regions of the left and right breast. We propose that a successful automated system can be developed using the same principle. The system is explained with the aid of the diagram in figure 2. Firstly, tissue types in the digitised mammogram are segmented to form anatomically homogeneous fat or non-fat regions. Asymmetry is then detected by comparing various features of non-fat regions in the left and right mammogram. Finally, the evidence from these comparisons is combined, in order to classify the case as normal or abnormal, and to locate any suspicious regions. The main advantage of this approach over conventional methods is that the non-fat regions are extracted from the mammogram and com- 
pared directly, so asymmetry measurements are likely to be more robust than those obtained using problematic breast alignment procedures. It is also possible to compare the shape of the regions, and thus recognize certain signs of architectural distortion which were not available to previous methods.

Work on the segmentation of mammograms into fat and non-fat regions has been published previously [8]. The results showed that Laws' texture energy [9] was the most successful segmentation method tested, on average correctly classifying $80 \%$ of the breast area in a 40 mammogram data set.

The following sections describe the development of the remaining components of our system in detail: asymmetry measurement and classification.

\section{Asymmetry cues}

The aim of these experiments is to identify successful methods for the detection of asymmetry between non-fat regions of the left and right breast.

\subsection{Materials and methods}

Two types of region features can be used in the comparison of non-fat regions: global features, which use a single value to describe some property of the whole region; and local features, which describe each location in the region individually. To compare global features of regions, it is only necessary to measure the difference between two values. To compare local features, an alignment process must first be performed, so that the locations in each region correspond. The difficulty in creating such a correspondence between whole breasts was discussed previously, but it is likely that the problems in aligning smaller anatomically homogeneous regions would not be as severe. We have initially concentrated on global features.

A data set of 104 screening mammogram pairs was used for these experiments, digitised at $0.2 \mathrm{~mm} / \mathrm{pixel}$ and 8 bits. The 57 asymmetric cases were further classified as densities (26), distortions (16) and masses (13). A radiologist annotated the non-fat regions as a temporary replacement for the automated breast segmentation method, to exclude the effects of segmentation errors from our analysis of comparison methods.

\subsubsection{Shape}

Cancer is known to distort the shape of anatomical structures, in some cases even before a mass is detectable [11]. By comparing the shape of gland discs from left and right mammograms, we may be able to detect both this architectural distortion, and any masses affecting the disc boundaries. Shape measurements can only be calculated from single closed regions, so for these experiments we assumed that the largest nonfat region in each breast was the gland disc, and discarded all smaller regions. An alternative approach would be to use the convex hull of non-fat regions. We calculated a shape measurement of left and right gland discs, and used the difference as a measure of asymmetry. No registration of the gland discs was required.

After exploring a number of shape measurement techniques [eg 12,13], we found that circularity and Fourier features achieved some degree of discrimination between normal and abnormal cases. We did not consider model-based shape descriptors, as they 
rely on the identification of reliable landmark points and minimal (or predictable) variation.

The Fourier transform was calculated by treating the coordinates of the gland disc contour as a one-dimensional complex function, always starting and ending at the pixel closest to the nipple. Veillon describes several features derived from the Fourier transform, including contour energy, roughness and deviation [13]. These features are sensitive to shape rotation, enabling similarly shaped regions at different orientations to be rated as asymmetric; this is a desirable property in mammographic analysis.

\subsubsection{Brightness distribution}

A limitation of shape measurements is their inability to detect masses which are not near the border of the gland disc. This section describes two techniques for comparing the distribution of brightness within non-fat regions: moments and transportation.

In operations research, the transportation problem is that of minimizing the cost of transporting goods from many warehouses to many shops, each having different availabilities or requirements. There is a cost associated with each route, so the optimal solution must fulfil shop requirements using local warehouses where possible. We decided to use this analogy to calculate the minimal cost of matching regions from left and right mammograms. This cost could then be considered a measure of asymmetry between the regions. Transportation can be formulated as a linear programming problem [14]:

$$
\begin{array}{lll}
\text { minimise } & t=\sum_{s, d} c_{s d} q_{s d} & \\
\text { subject to } & \sum_{d} q_{s d}=A_{s} & \text { for all } s \\
& \sum_{s}^{s} q_{s d}=R_{d} & \text { for all } d \\
q_{s d} \geq 0 & \text { for all } s, d \\
& \sum_{s} A_{s}=\sum_{d} R_{d} &
\end{array}
$$

where $t$ is the total transportation cost, $q_{s d}$ is the quantity transported from source $s$ to destination $d, c_{s d}$ is the cost per unit transportation from source $s$ to destination $d, A_{s}$ is the availability at source $s$, and $R_{d}$ is the requirement at destination $d$. If the total availability and requirement are unequal, then a dummy site is added to satisfy the final constraint.

In our model, the domain of source sites is represented by non-fat pixels in the left breast, and destination sites by non-fat pixels in the right breast. Pixel values represent the availability or requirement at each site. The cost of transportation between sites should be a measure of distance, so as to minimize the warping required for a match. As the source and destination sites are on different mammograms, the domains are aligned (with respect to nipple locations) to allow distances to be calculated. We found that by reducing the size of the images, the transportation problem could be solved rapidly, with only a minor effect on results.

\subsubsection{Topology}

Further diagnostic information can be extracted from the topology of non-fat regions within the breast. For instance, a small region isolated from the gland disc is suspi- 
cious, especially if it is in an unusual location, such as between the gland disc and the pectoral muscle. So far the only topology features we have considered are area and binary moments, which compare the distribution of non-fat tissue in left and right mammograms. The method is the same as for brightness moments, except that the image function is defined to be 1 for non-fat coordinates, and 0 elsewhere. A rulebased expert system would be appropriate for providing further asymmetry evidence from topology.

\subsection{Results}

For each asymmetry feature described above, observations were generated from the data set of mammogram pairs. The variance-ratio test (or $F$-test) was used to find the best features for discriminating between normal and abnormal groups. This test measures the degree of separation of two group means, as the ratio of between-group to within-group variances. The different asymmetry types were considered both together and separately, each time with the same set of normal cases. Six features were found to be effective discriminators (table 1).

\begin{tabular}{|l|c|ccc|}
\hline Feature & $\begin{array}{c}\text { All } \\
\text { asymmetries }\end{array}$ & Density & $\begin{array}{c}\text { Type of asymmetry } \\
\text { Distortion }\end{array}$ & Mass \\
\hline Contour & 3.8 & 5.8 & 1.0 & 0.2 \\
Deviation & 3.8 & 7.8 & 0.0 & 0.9 \\
Roughness & 1.3 & 2.6 & 0.0 & 0.7 \\
Circularity & 0.3 & 0.0 & 5.6 & 0.0 \\
Transportation & 6.7 & 6.6 & 4.3 & 4.6 \\
Area & 4.6 & 0.1 & 7.4 & 6.3 \\
\hline
\end{tabular}

Table 1. Variance-ratio test for measuring the discriminating power of asymmetry features

\subsection{Discussion}

The strongest shape features are derived from the fourier transform, which is a powerful method for describing the significant deviations of the gland disc contour. The best brightness feature is transportation, and non-fat area was a useful topology feature. It can be seen that different features are successful in discriminating the distinct types of asymmetry from normal cases. This suggests that asymmetry should be detected using separate classification schemes for each asymmetry type.

We are currently investigating texture as a further global feature, to aid recognition of architectural disturbance. Brightness, density and texture will also be considered for the local comparison of non-fat regions. Another question being considered is whether the left and right breasts should be corrected for size and shape differences before the non-fat regions are compared. Although this might eliminate differences due to breast compression, warping could introduce unnatural distortion of internal structures, especially if the breasts are actually different in size. Further analysis of the transportation solution and moment values should allow positional information for asymmetries to be generated. 


\section{Classification}

The purpose of this work is to classify mammogram pairs into normal and abnormal categories, based on their degree of symmetry.

\subsection{Materials and methods}

Our approach relies on many automated measurements, each providing evidence about the presence of asymmetry. As the signs of asymmetry are subtle, the evidence supplied by any single measurement is likely to be too weak for classification purposes. However, combination of evidence may improve discrimination considerably. This technique was used successfully by Astley and Taylor [15] for the detection of microcalcifications.

We tested three classifiers for the combination of asymmetry evidence: linear discriminant, quadratic discriminant and k-nearest-neighbour ( $\mathrm{kNN}$ ) [16]. We found that the linear classifier gave the best performance for this application. Discriminant classifiers create decision boundaries in feature space for classifying observations into groups. Optimal decision boundaries are calculated from the means and covariances of training observations of each group. These classifiers assume normal distributions for the features, though they are robust to minor violations.

Three data sets were derived from the 104 mammograms, each containing examples of only one asymmetry type, and all of the normal cases. In this way we were able to develop and test separate classification schemes for densities, distortions and masses. Each classifier used the six features described above, and was trained and tested on a leave-one-out basis. No attempt has yet been made to optimise the classifier by adjusting the prior group probabilities, which are currently assumed to be equal.

\subsection{Results}

Table 2 presents the classification performance obtained when considering asymmetry types separately and together. The final column indicates the performance of the separate-type classifiers on the whole data set.

\begin{tabular}{|l|c|ccc|c|}
\hline & Asymmetries & \multicolumn{3}{|c|}{ Type of asymmetry } & Asymmetries \\
& together & Density & Distortion & Mass & separately \\
\hline Hit rate & 53 & 68 & 56 & 76 & 64 \\
False alarm rate & 23 & 19 & 19 & 14 & 17 \\
\hline Accuracy & 64 & 76 & 75 & 84 & 72 \\
\hline
\end{tabular}

Table 2. Classification results using linear discriminant classifier, with equal prior probabilities and leave-one-out training

\subsection{Discussion}

Use of the three separate classification schemes in combination is clearly more effective than the original scheme in which all types of asymmetry were considered together. Although the combined hit rate is as yet only moderately high, we believe that further optimisation of our methods will improve the results to a clinically acceptable extent. We now have evidence that prompting, in which radiologists' attention is drawn to abnormalities detected by computer, may still be effective at lower hit rates 
provided that the false alarm rate is kept low [17]. In this light, our results are very encouraging.

\section{Conclusions}

The detection of breast asymmetry is a difficult problem, even for experienced radiologists. The ill-defined, variable and subjective nature of asymmetric visual cues makes the problem even harder to define in terms of computer vision techniques. Nevertheless, we must focus our effort toward such challenging problems if computers are to provide useful assistance to radiologists. We have developed a new automated approach to the detection of breast asymmetry, modelled on the regional comparison identified in radiologists' technique. Digitised mammograms are segmented into regions of fat and non-fat tissue; non-fat regions in the left and right breast are compared using shape, brightness and topology features. Shape is used to detect architectural distortion of the gland disc; brightness distribution detects masses and focal densities; and topological rules specify other suspicious asymmetries. As breast registration is not required by this approach, we believe that it will eventually be possible to detect smaller and more subtle abnormalities than those detected by conventional methods.

We have developed a texture analysis method for segmenting the breast image into anatomical regions, on average classifying $80 \%$ of the breast area correctly. We need to combine several texture and brightness features to improve this accuracy, and enforce spatial constraints on the segmentation to produce contiguous regions suitable for asymmetry analysis. Non-fat regions of the left and right breast have been compared using shape and brightness features from the literature. We adopted a divideand-conquer strategy: separate data sets for each distinct type of asymmetry were assembled, so that the best features for each type could be identified and combined to improve the overall detection of asymmetry.

The asymmetry evidence from different features has been combined to achieve $72 \%$ correct classification of a data set of 104 very subtle mammographic cases. We are now exploring methods for improving the detection of asymmetry, and for generating locations of potential abnormalities, with the aim of producing a technique suitable for prompting radiologists in a computer-aided diagnosis system for breast screening.

\section{Acknowledgements}

This research is funded by SERC and IBM UK Scientific Centre. The authors would particularly like to thank Drs Caroline Boggis and Mary Wilson, consultant radiologists at The Nightingale Centre, Withington Hospital, for their invaluable participation in discussions and experiments. 


\section{References}

1. Yin F-F, Giger ML, Doi M, Metz CE, Vyborny CJ, Schmidt RA. Computerized detection of masses in digital mammograms: analysis of bilateral subtraction images. Medical Physics 18:955-963, 1991.

2. Kimme C, O'Loughlin BJ, Sklansky J. Automatic detection of suspicious abnormalities in breast radiographs. Data Structures, Computer Graphics and Pattern Recognition, Academic Press, New York, 1975.

3. Lau T-K, Bischof WF. Automated detection of breast tumours using the asymmetry approach. Computers and Biomedical Research 24:273-295, 1991.

4. Hand W, Semmlow JL, Ackerman LV, Alcorn FS. Computer screening of Xeromammograms: a technique for defining suspicious areas of the breast. Computers and Biomedical Research 12:445-460, 1979.

5. Egan RL. Breast imaging - diagnosis and morphology of breast diseases. WB Saunders, London, 1988.

6. Boggis CRM, Hufton AP, Asbury DL et al. Manchester breast screening manual. Withington Hospital, 1990.

7. Tabar L, Dean PB. Teaching atlas of mammography. Thieme, New York, 1985.

8. Miller PI, Astley SM. Classification of breast tissue by texture analysis. Image and Vision Computing 10:277-282, 1992.

9. Laws KI. Textured image segmentation. Report 940, Image Processing Institute, University of Southern California, Los Angeles, 1980.

10. Miller PI, Astley SM. Detection of breast asymmetry using anatomical features. Proceedings of SPIE Biomedical Image Processing and Visualisation, 1993.

11. Kopans DB. Breast imaging. Lippincott, Philadelphia, 1989.

12. Marshall S. Review of shape coding techniques. Image and Vision Computing 7:281-294, 1989.

13. Veillon F. Study and comparison of certain shape measures. Signal Processing 11:81-91, 1986.

14. Hartley R. Linear and nonlinear programming. Ellis Horwood, Chichester, 1985.

15. Astley SM, Taylor CJ. Combining cues for mammographic abnormalities. Proceedings of the British Machine Vision Conference, pp. 253-258, 1990.

16. Therrien CW. Decision, estimation, and classification: an introduction to pattern recognition and related topics. John Wiley, New York, 1989.

17. Astley S, Hutt I et al. Automation in mammography: computer vision and human perception. Proceedings of SPIE Biomedical Image Processing and Visualisation, 1993. 